For this reason the usefulness of $\alpha$-MPT as a routine addition to the pre-operative regime appears to be further justified.

The widespread $\mathrm{T}$ wave abnormalities seen in this patient were in keeping with the changes documented elsewhere (Van Vliet et al., 1966; Pelkonen and Pitkänen, 1963). These changes persisted in the absence of hypertension but gradually resolved following the second adrenalectomy.

The advantage of having reduced concentrations of both circulating and tumour catecholamines at the time of operation, and the possibility of preventing a myocarditis, would seem to warrant further investigation of $\alpha$-MPT (in conjunction with adrenergic blocking agents) in the routine pre-operative management of phaeochromocytoma.

\section{Acknowledgments}

We wish to thank the following for their help in this case: Mr D. Pratt (surgeon), Dr M. Roberts (anaesthetist), Dr J. M. Glanville (radiology), Dr G. Hardy (pathology), Dr J. Terry (medical physics), Dr C. Hayter (isotope studies), Mr G. Clough (biochemist) and the staff of the Chemical Pathology Departments of Leeds General Infirmary and St James's Hospital, Leeds. We are also indebted to Dr A. Rhymer of
Merck-Sharpe and Dohme Ltd for his assistance and for supplying $\alpha$-methyl-p-tyrosine.

\section{References}

Engelman, K., Horwitz, D., Jequier, E. \& Sjoerdsma, A. (1968a) Biochemical and pharmacological effects of $\alpha$ methyltyrosine of man. Journal of Clinical Investigation, 47, 577.

Engelman, K., Jequier, E., Udenfriend, S. \& Sjoerdsma, A. (1968a) Metabolism of $\alpha$-methyltyrosine in man. Journal of Clinical Investigation, 47, 568.

KLINE, I. (1961) Myocardial alterations associated with pheochromocytomas. American Journal of Pathology, 38, 539.

Pelkonen, R. \& Pitkänen, E. (1963) Unusual electrocardiographic changes in phaeochromocytoma. Acta medica scandinavica, 173, 41 .

Ross, E.J., Prichard, B.N.C., Kaufman, L., Robertson, A.I.G. \& HARries, B.J. (1967) Preoperative and operative management of patients with phaeochromocytoma. British Medical Journal, 1, 191.

Sjoerdsma, A., Engelman, K., Spector, S. \& Udenfriend, S. (1965) Inhibition of catecholamine synthesis in man with $\alpha$-methyltyrosine, an inhibitor of tyrosine hydroxylase. Lancet, ii, 1092.

VAn Vliet, P.D., Burchell, H.B. \& Titus, J.L. (1966) Focal myocarditis associated with pheochromocytoma. New England Journal of Medicine, 274, 1102.

\title{
Caroli's disease with intrahepatic gall-stones and Salmonella infection
}

\author{
A. N. HAMLYN \\ B.Sc., M.B., M.R.C.P. \\ O. F. W. JAMES \\ A. P. Douglas \\ B.Sc., M.D., M.R.C.P., M.R.C.P.(Ed) \\ M.A., M.R.C.P. \\ M. I. LAVELLE \\ M.B., F.F.R.
C. W. Venables
M.S., F.R.C.S. \\ Departments of Gastroenterology and Radiology, Newcastle University Hospitals
}

\begin{abstract}
Summary
At operation for small bowel intussusception, a 26year-old man was found to have an enlarged liver and spleen. Subsequent investigations suggested bile passage infection associated with numerous intrahepatic gall-stones but symptomatic cholangitis did not present until 5 months later. Retrograde cholangiography showed cavernous ectasia of the bile ducts which contained gall-stones.
\end{abstract}

Correspondence: Dr A. N. Hamlyn, Department of Gastroenterology, Royal Victoria Infirmary, Queen Victoria Road, Newcastle Upon Tyne NE1 4LP.
Bile aspirated from the liver contained Salmonella agona. Despite treatment with ampicillin he remains a chronic Salmonella carrier. The importance is stressed of searching for enteric organisms in the bile and faeces of patients with chronic biliary disease especially when associated with cholelithiasis.

\section{Introduction}

Chronic calculous disease is a well known concomitant of the typhoid carrier state. More rarely these two conditions are associated with the extremely uncommon Caroli's disease (Waldram, 


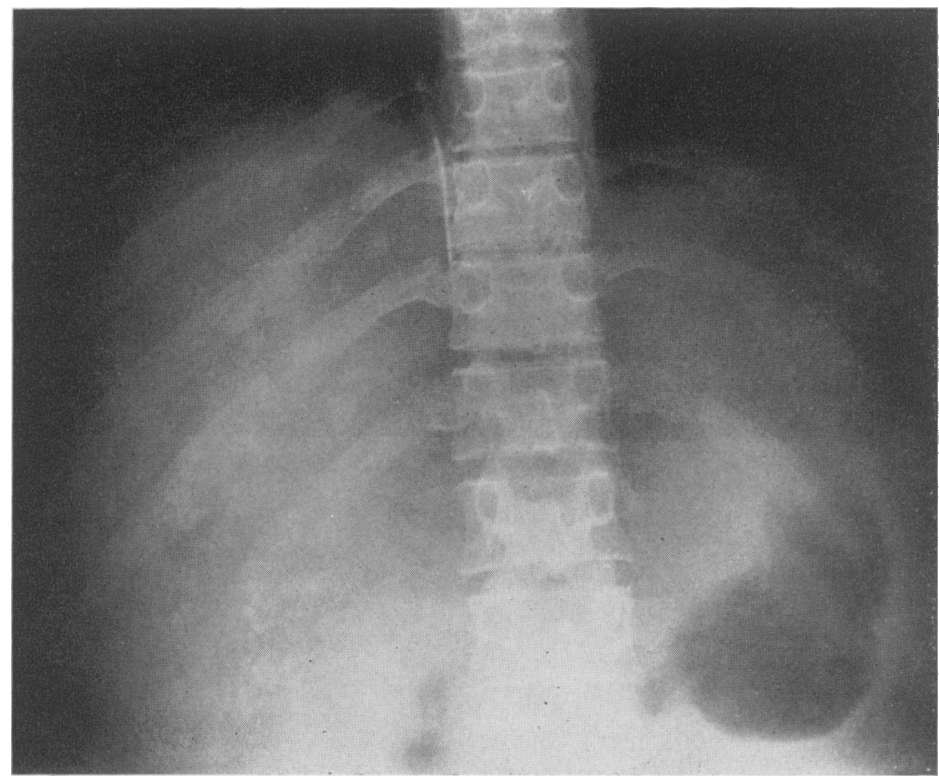

Fig. 1. Plain abdominal X-ray. There are numerous areas of calcification in the liver area.

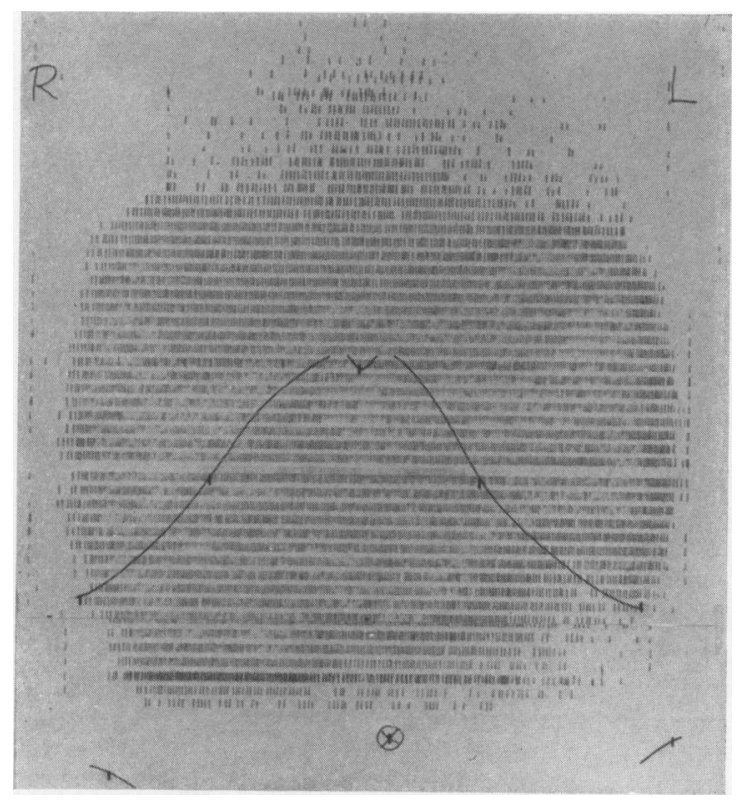

FIG. 2. ${ }^{99} \mathrm{Tc}$ colloid liver scan. Numerous cystic filling defects are present throughout the liver.

Vahrman and Williams, 1975). This report describes such a case with $S$. agona cultured from bile and faeces. He has now become a chronic carrier.

\section{Case report}

In May 1974 a 26-year-old labourer was admitted to hospital with severe stomach pain, rigid abdomen and shock. At laparotomy a portion of intussuscepted small bowel was removed. At the same time an irregularly enlarged liver was noticed. He made an uneventful recovery but 5 months later was readmitted with symptoms of cholangitis and septicaemia. At that stage, liver and spleen were palpable but there were no stigmata of chronic liver disease.

Investigations showed normal white cell counts with neutrophilia. Blood cultures remained sterile. Serum bilirubin $0.5 \mathrm{mg} / \mathrm{dl}$, alkaline phosphatase 163 i.u./l (normal 20-90), aspartate transaminase 29 i.u./l (normal 4-20), serum albumin $2 \cdot 2 \mathrm{~g} / \mathrm{dl}, \mathrm{HB}_{\mathrm{s}} \mathrm{Ag}$ negative, smooth muscle antinuclear and mitochondrial antibody absent. Blood urea was normal. Plain abdominal X-ray (Fig. 1) showed numerous areas of calcification in the liver. ${ }^{99} \mathrm{Tc}$ (Fig. 2) and ${ }^{67} \mathrm{Ga}$ liver scan demonstrated intrahepatic filling defects thought to be cysts rather than abscesses. Aspiration needle biopsy of the liver by the Menghini technique resulted in the recovery of small calculi and bile from which $S$. agona was cultured. This organism was also found in the faeces. The Widal test was positive to $S$. typhi 'O' to a titre of $1 / 250$. Liver histology confirmed the absence of cirrhosis but portal areas were enlarged and connected by well developed collagenous septa. There was bile ductule proliferation and a microcalculus was seen in one of these. 


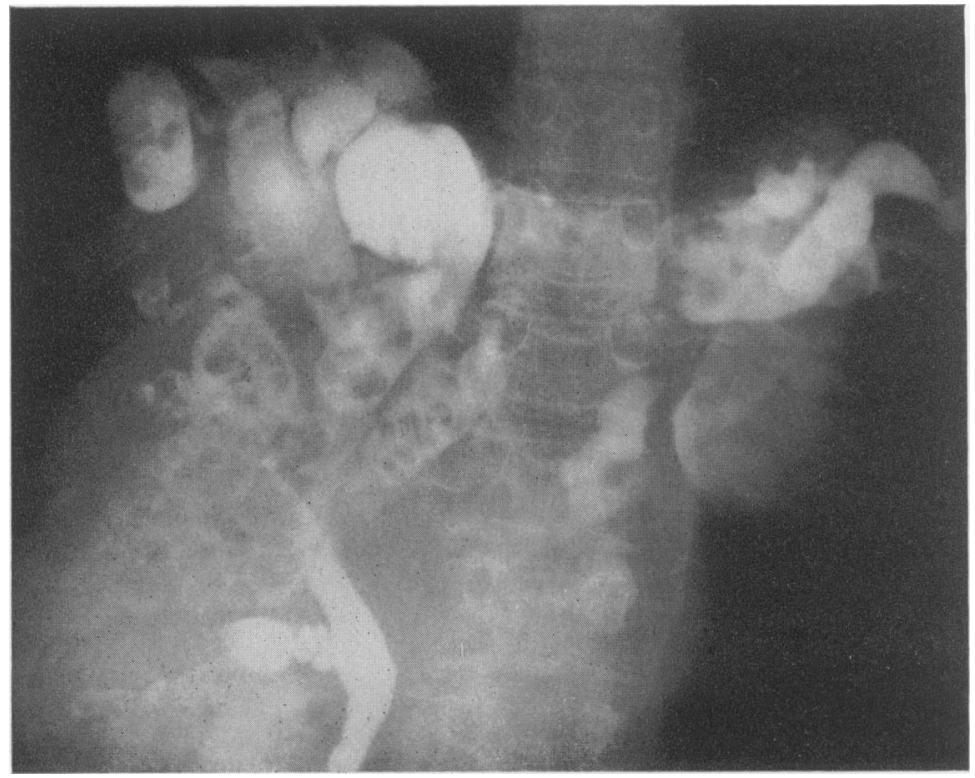

FIG. 3. Endoscopic retrograde cholangiography shows cavernous dilatation of the intrahepatic bile ducts. Numerous filling defects due to gall-stones are also present.

Endoscopic retrograde cholangiography (Fig. 3) demonstrated cystic dilatation of the intrahepatic biliary tree with numerous gall-stones. Barium swallow failed to show varices. Intravenous urography suggested the presence of renal cysts.

\section{Treatment and progress}

In view of the severity of the patient's illness, intravenous ampicillin therapy was started and, $48 \mathrm{hr}$ later, intramuscular gentamicin. After an episode of collapse and hypotension, he recovered only to experience a further mild cholangitic episode responding to the same antibiotic combination a few days later. He eventually recovered and is now asymptomatic. Nevertheless he continues to excrete Salmonella organisms and to exhibit cholestatic liver function tests.

\section{Discussion}

The description of this case fits that of communicating ectasia of intrahepatic bile ducts as first recorded by Caroli (Caroli and Couineaud, 1958). In this there is segmental dilatation of the intrahepatic biliary tree, predisposition to calculous biliary disease and cholangitis, with absence of cirrhosis and portal hypertension. It differs from the one previous description of Salmonella infection with Caroli's disease in that blood cultures for the organism re- mained consistently negative. In addition, isolation of $S$. agona was achieved by needle biopsy of the liver and intrahepatic gall-stones were present.

Although one review (Mujeted, Glenn and Evans, 1971) cites over $50 \%$ of Caroli's disease patients as possessing biliary calculi and many of these are infected, the organisms involved are usually common gut commensals. Despite the association of cholelithiasis with the typhoid carrier state, isolation of Salmonella from bile or faeces seems to be a rarity in Caroli's disease.

This case presents great problems of management. Therapeutic levels of ampicillin are not achieved in gall-stones (Scioli, Fiorentino and Sasso, 1972). Gentamicin reaches the bile ducts with difficulty in obstructive jaundice (Smithives, Hyams and Patiol, 1971) and chloramphenicol can be hepatotoxic under these circumstances (Lazar, 1966). The emphasis, therefore, is on characterizing all cases of infected chronic biliary obstruction and excluding Salmonella infection of the bile passages by routine culture of bile, liver biopsy material and faeces. Such cases are very likely to be poor operative risks for removal of gall-stones and will fail to respond to antibiotics. They will then become significant public health risks and require careful medical and community health management. This is particularly so in the care of Caroli's disease which is not amenable to surgery and may run a very protracted course. 


\section{Acknowledgments}

A.N.H. was supported by a Newcastle University Hospitals' Scientific and Research Committee Grant.

\section{References}

CARoli, S. \& Couineaud, C. (1958) Une affection nouvelle, sans doute congénitale, des voies biliaires; la dilatation kystique unilobaire des canaux hépatiques. Semaine des Hôpitaux de Paris, 14, 136.

LAZAR, A.J. (1966) Cirrhosis, Gram-negative sepsis and chloramphenicol toxicity. American Journal of Gastroenterology, 45, 29.
Mujeted, L., Glenn, F. \& Evans, J.A. (1971) Communicating cavernous ectasia of the intrahepatic ducts (Caroli's disease). American Journal of Roentgenology, 113, 21.

Scioli, C., Fiorentino, F. \& SASso, G. (1972) Treatment of Salmonella typhi carriers with intravenous ampicillin. Journal of Infectious Diseases, 125, 170.

Smithives, T., Hyams, P.J. \& Patiol, J.J. (1971) Gentamicin and ampicillin in human bile. Journal of Infectious Diseases. 124, 106.

WALdRAM, R.M.R., VAHRMAN, J. \& Williams, R. (1975) Salmonella heidelberg infection in Caroli's syndrome. Gastroenterology. 68, 151.

\title{
Systemic mastocytosis-central nervous system features and lymphographic demonstration of lymph node involvement
}

\author{
B. W. HANCOCK \\ M.B., Ch.B., D.C.H., M.R.C.P. \\ P. DANDONA \\ M.B., B.S., M.R.C.P., D.Phil. (Oxon) \\ D. C. Cumberland* \\ M.B., Ch.B., D.M.R.D., F.F.R. \\ J. A. JARRATT $\dagger$ \\ M.B., Ch.B., M.R.C.P. \\ Academic Division of Medicine, *Department of Radiology, The Royal Hospital, Sheffield S1 3SR, and \\ †Department of Clinical Neurophysiology, Fulwood Annexe, Sheffield S10 3TD
}

\begin{abstract}
Summary
Systemic mastocytosis is uncommon. A patient is reported with many of the characteristic lesions of this disorder but with two features not before describedthe occurrence of mixed pyramidal and neuromyopathic neurological signs and the demonstration of lymph nodes with abnormal architecture by lymphography.

\section{Case report}

A female patient (Fig. 1) presented in 1967 aged 54 years with cutaneous urticaria pigmentosa (confirmed by skin biopsy). In 1972 splenic enlargement was noted but was not investigated. She was admitted to hospital in May 1974 with a history of ill health and weight loss of $35 \mathrm{~kg}$ over the previous year. On examination she was emaciated, anaemic and showed characteristic skin lesions of urticaria pigmentosa. Liver and spleen were each enlarged to $12 \mathrm{~cm}$ below costal margins. Extensive investigations gave these abnormal results: haemoglobin $9 \cdot 5 \mathrm{~g} / 100$ $\mathrm{ml}$ (red cells microcytic and normochromic); leucocyte count $8,000 / \mathrm{mm}^{3}(10 \%$ monocytes $)$; platelet count $60,000 / \mathrm{mm}^{3}$; ESR (Westergren) $45 \mathrm{~mm} / \mathrm{hr}$; circulating heparin detected $(0 \cdot 3 \mathrm{u} . / \mathrm{ml}$ by specific
\end{abstract}

heparin assay); red cell half-life (after ${ }^{51} \mathrm{Cr}$-labelling) reduced to 14.6 days; serum iron and total iron binding capacity low ( 9 and $48 \mu \mathrm{mol} / 1$ respectively); bone marrow and bone infiltrated by mast cells; serum alkaline phosphatase elevated $(410 \mathrm{u}$./l) serum 5 nucleotidase elevated to $38 \mathrm{u} . / 1$, serum hepatocellular enzymes uniformly elevated; skeletal survey showed characteristic sclerotic changes; and bipedal lymphogram demonstrated abnormal iliac and paraaortic lymph nodes (see below).

At this stage the patient was treated with prednisolone ( $20 \mathrm{mg}$ daily). The size of the liver and spleen diminished; the ESR fell to $16 \mathrm{~mm} / \mathrm{hr}$ and haemoglobin rose to $12 \mathrm{~g} / 100 \mathrm{ml}$. However, over the next few months she developed weakness of arms and legs which progressed even though the dosage of prednisolone was reduced to $5 \mathrm{mg}$ daily. There was also episodic urinary and faecal incontinence. Examination revealed marked proximal weakness in addition to bilateral diffuse loss of power in the pyramidal distribution. The deltoid, biceps brachii, quadriceps femoris and hamstring muscles were wasted but no fasciculation was seen. Some incoordination of the legs was present, probably attributable to her weakness. The jaw jerk and other 